\title{
Ginkgo biloba extract protects diabetic rats against cerebral ischemia-reperfusion injury by suppressing oxidative stress and upregulating the expression of glutamate transporter 1
}

\author{
MIAO YAN ${ }^{1,2 *}$, MEI LI $^{1 *}$, SHULING GU $^{3}$, ZHENG SUN $^{3}$, TENGFEI MA $^{3}$ and XING MA ${ }^{3}$ \\ ${ }^{1}$ Jiangsu Key Laboratory of New Drug Research and Clinical Pharmacy, Xuzhou Medical University, Xuzhou, Jiangsu 221004; \\ ${ }^{2}$ Medicine and Pharmacy Research Center, Binzhou Medical University, Yantai, Shandong 264003; \\ ${ }^{3}$ Department of Pharmacology, School of Pharmacy, Xuzhou Medical University, Xuzhou, Jiangsu 221004, P.R. China
}

Received May 21, 2019; Accepted January 14, 2020

DOI: $10.3892 / \mathrm{mmr} .2020 .10990$

\begin{abstract}
The current study aimed to evaluate the neuroprotective effect of Ginkgo biloba extract $(G b \mathrm{E})$ on the progression of acute cerebral ischemia-reperfusion injury in diabetic rats, and to determine the molecular mechanism associated with this effect. Streptozotocin (STZ) induced diabetic rats were pretreated with $G b \mathrm{E}(50,100$ and $200 \mathrm{mg} / \mathrm{kg} /$ day; intragastric) for 3 weeks. During this period, body weight changes and fasting blood glucose levels were assessed each week. Following pretreatment, rats were subjected to suture occlusion of the middle cerebral artery for $30 \mathrm{~min}$, which was followed by $24 \mathrm{~h}$ of reperfusion. Neurological deficits were subsequently evaluated at 2 and $24 \mathrm{~h}$ following reperfusion. Rats were sacrificed after $24 \mathrm{~h}$ reperfusion, and infarct volume and S100B content were measured to evaluate the neuroprotective effect of $G b \mathrm{E}$. The results of the present study demonstrated that $G b \mathrm{E}$ pretreatment improved neurological scores, and reduced cerebral infarct volume and $\mathrm{S} 100 \mathrm{~B}$ content. Oxidative stress markers, including glutathione (GSH) and superoxide dismutase (SOD) were increased, and malondialdehyde (MDA) contents were reduced following $G b \mathrm{E}$ treatment. The levels of p-Akt, p-mTOR and glutamate transporter 1 (GLT1) were observed to be increased in $G b \mathrm{E}$-pretreated rats. These results indicated that $G b \mathrm{E}$ pretreatment may serve a protective role against cerebral ischemia-reperfusion injury in diabetic rats by inhibiting oxidative stress reaction, upregulating the expression of Akt/mTOR and promoting GLT1 expression. In conclusion, the current study revealed the protective role and
\end{abstract}

Correspondence to: Professor Mei Li, Jiangsu Key Laboratory of New Drug Research and Clinical Pharmacy, Xuzhou Medical University, 209 Tongshan Road, Xuzhou, Jiangsu 221004, P.R. China E-mail: limei0927@gmail.com

*Contributed equally

Key words: Ginkgo biloba extract, diabetes, cerebral ischemia, oxidative stress, glutamate transporter 1 molecular mechanisms of $G b \mathrm{E}$ in diabetic rats with cerebral ischemia-reperfusion injury, and may provide novel insight into the future clinical treatment of this condition.

\section{Introduction}

Diabetes mellitus is a complex metabolic syndrome, which significantly affects systemic and cerebral vasculature (1). Chronic and uncontrolled diabetes mellitus is characterized by a persistent elevation in blood glucose, which is in association with a number of long-term complications, including ischemic stroke (1). Diabetes is an independent risk factor for ischemic stroke (2). Diabetes exacerbates cerebral ischemia injury in experimental and clinical stroke subjects by accelerating neuronal damage and increasing infarct volume (3-5). Patients with diabetes exhibit double the risk of ischemic stroke compared with people without diabetes, after correction for other risks, and these individuals are predicted to exhibit increased morbidity and mortality $(6,7)$. However, the cellular and molecular mechanisms by which hyperglycemia is associated with ischemic brain damage have not been fully determined.

Oxidative stress serves a pivotal role in the development of microvascular and macrovascular diabetes complications (8). The overproduction of reactive oxygen species (ROS), which is induced by hyperglycemia, is a mediator of tissue damage that occurs during diabetes, and can lead to cerebral dysfunction $(8,9)$. The brain is vulnerable to radical-mediated attack due to its limited antioxidant defenses (10). When the redox balance is impaired, free radicals and oxidative stress-associated mechanisms can cause cell injury and necrosis (11). During periods of oxidative stress, Akt/mTOR pathways are closely integrated and have been revealed to directly determine cell fate (12-14). Research has demonstrated that the Akt/mTOR signaling cascade serves an important role in the onset and progression of cerebral ischemia injury (13). A number of agents that increase the phosphorylation levels of Akt and mTOR have been demonstrated to reduce brain injury in stroke models $(15,16)$. A previous study has also indicated that lentiviral-mediated overexpression of cAkt can protect against stroke-induced neuronal injury in vivo and in vitro, and 
mTOR inhibition with rapamycin can block these protective effects (17).

Excitotoxicity, which is induced by the overactivation of glutamate, has been identified as a key factor in the pathogenesis of cerebral ischemia (18). GLT1, which is predominantly located on astrocytes, is responsible for up to $90 \%$ of glutamate clearance to maintain glutamate homeostasis in adult brain tissue (19). However, the downregulation or dysfunction of GLT1 following ischemia leads to the accumulation of extracellular glutamate and neuronal death (20). It has been demonstrated that GLT1 knockdown exacerbates the neuronal death and neurological deficit in rats with middle cerebral artery occlusion (MCAO) (21). Recent evidence has demonstrated that mTOR is a downstream target of the PI3K/Akt pathway, which regulates GLT1 expression (22). mTOR complex1 (mTORC1) and mTOR complex2 (mTORC2) are associated with GLT1 expression (23), and oxidative stress and excitotoxic mechanisms have been suggested to operate in tight conjunction to induce irreversible damage of brain tissue (24). Chen et al (25) proposed that glutamate-mediated excitotoxicity with oxidative stress fulfill the 'two-hit' hypothesis that accelerates neurodegeneration. Therefore, the current study hypothesized that oxidative stress causes the downregulation of Akt/mTOR signaling, and mTOR participates in the downregulation of GLT1, which can lead to further excitotoxicity, and eventually exacerbate diabetic ischemic stroke.

Ginkgo biloba extract $(G b \mathrm{E})$ is a standardized mixture that is extracted from Ginkgo biloba leaves, containing 22-27\% Ginkgo flavone glycosides (myricetin, quercetin, kaempferol and isorhamnetin) and 5-7\% terpene lactones (ginkgolide A, B, C and bilobalides) (26). $G b \mathrm{E}$ has been used as a therapeutic agent for a number of cardiovascular and neurological diseases $(27,28)$. Although the exact mechanism is unclear, an accumulation of evidence has demonstrated that $G b \mathrm{E}$ exhibit a number of benefits, including improving hemodynamics, inhibiting the platelet-activating factor, scavenging ROS and relaxing vascular smooth muscles (29). These results demonstrate the pharmacological use of $G b \mathrm{E}$ for the treatment of diabetic ischemic stroke. Recent studies have demonstrated that $G b$ E protects against a number of diabetic complications, including diabetic cataract (30), diabetic nephropathy (31) and diabetic cardiomyopathy (32). However, the effect of $G b \mathrm{E}$ on diabetic ischemic stroke is yet to be determined. Therefore, the present study was designed to evaluate the protective effect and its possible mechanism of action in diabetic rats with cerebral ischemia-reperfusion injury.

\section{Materials and methods}

Animals. Adult male Sprague-Dawley rats (8-10 weeks old; 180-220 g) were obtained from the Laboratory Animal Center of Xuzhou Medical University, (license no. SCXK2007-2005; Xuzhou, China), where an SPF level laboratory was founded, as authorized by the Jiangsu province government. All animals were maintained at a constant temperature of $25 \pm 2^{\circ} \mathrm{C}$ under a $12 / 12 \mathrm{~h}$ light/dark cycle. Rats were allowed free access to food and water ad libitum. Animal experiments were conducted in accordance to the principles provided by National Institute of Health (NIH) Guideline for the Care and use of Laboratory Animals. The approval to proceed with this experiment was issued by the Animal Ethics Committee of Xuzhou Medical University which also conforms to the Guidelines for Ethical Conduct in the Care and Use of Animals. All efforts were made to prevent unavoidable pain and distress when the approved endpoint is reached, no animal death occurred during this study. Euthanasia should result in rapid loss of consciousness, followed by respiratory and cardiac arrest and ultimate loss of all brain function. Death was confirmed after euthanasia and prior to disposal of the animal. Each rat was weighed weekly, while the measurement of blood glucose was performed using a glucometer via the tail vein (Nanjing Jianqiao Medical Device Co. Ltd.).

Drugs. GbE was used in the current study and is an extract of dried Ginkgo biloba leaves. GbE was obtained from Shaanxi Huike Botanical Development Co., Ltd. (Purity, $>98 \%$; cat. no. HK20121201). For administration in vivo, $G b \mathrm{E}$ was dissolved in $1 \% \mathrm{CMC}-\mathrm{Na}$ at concentrations of 10, 20 and $40 \mathrm{mg} / \mathrm{ml}$. Streptozotocin (STZ; cat. no. 0130) was purchased from Sigma-Aldrich; Merck KGaA. Nimodipine (cat. no. 120554), which was used as a positive control, was purchased from Yabao Pharmaceutical Group Co., Ltd. and suspended in $1 \% \mathrm{CMC}-\mathrm{Na}$ solution.

Diabetic model. The rats that were fasted overnight were subjected to a single intraperitoneal injection of $60 \mathrm{mg} / \mathrm{kg} \mathrm{STZ}$ that was freshly dissolved in $0.1 \mathrm{~mol} / 1$ cold citrate buffer at $\mathrm{pH}$ 4.3. Age-matched normal rats were injected with an equal volume of citrate buffer alone. Blood glucose was measured a period of one week after STZ injection. Rats with fasting blood glucose of $\geq 13.88 \mathrm{mmol} / \mathrm{l}$ were considered diabetic and included in the present study (30).

Focal cerebral ischemic model and grouping. Normal and diabetic rats were placed in the supine position on a heated pad and had a body temperature of $36.5-37.5^{\circ} \mathrm{C}$, which was monitored using a rectal thermometer. After being anesthetized with an intraperitoneal injection of $10 \%$ chloral hydrate $(300 \mathrm{mg} / \mathrm{kg})(33,34)$, rats were subjected to $30 \mathrm{~min}$ of middle cerebral artery (MCA) occlusion followed by $24 \mathrm{~h}$ of reperfusion. No signs of pain and peritonitis were observed following administration of $10 \%$ chloral hydrate. Sham operation and transient middle cerebral artery occlusion were performed as previously described (35). The right common carotid artery (CCA), external carotid artery, and internal carotid artery (ICA) were isolated. A nylon filament, which was purchased from Beijing Sunbio Biotech Co., Ltd., was subsequently introduced into the CCA lumen and gently advanced to the ICA until a slight resistance was felt.

A total of eighty rats were randomly divided into eight groups: The Con + sham group were nondiabetic and sham-operated rats (Con + sham group; $n=10)$; Con + ischemia-reperfusion (I/R) rats were nondiabetic and received I/R injury (Con + I/R group; $n=10$ ); diabetic and sham-operated rats were in the $\mathrm{STZ}+$ sham group (STZ + sham group, $\mathrm{n}=10)$; diabetic rats treated with ischemia-reperfusion injury were the STZ + I/R group (STZ + I/R group; $n=10)$. These aforementioned groups were administered the same volume of $1 \% \mathrm{CMC}$ solution for a period of three weeks. Before ischemia-reperfusion injury, the diabetic rats were administrated intragastrically with 


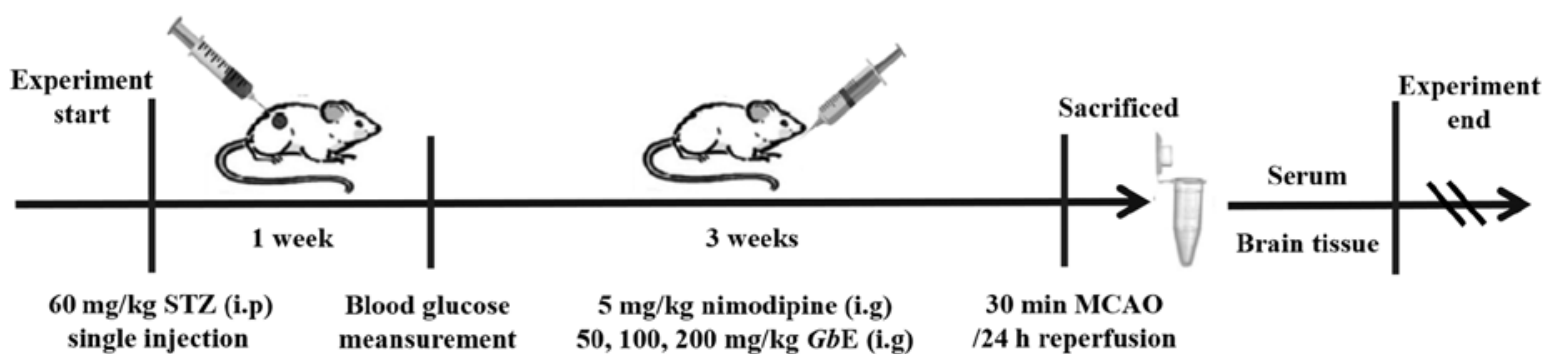

Figure 1. Schematic representation of the experimental protocol used. STZ, streptozotocin; GbE, Ginkgo biloba extract; MCAO, middle cerebral artery occlusion.

50,100 and $200 \mathrm{mg} / \mathrm{kg} G b \mathrm{E}$ for the GL group (low dose; $\mathrm{n}=10$ ), the GM group (moderate dose; $\mathrm{n}=10$ ) and the GH group (high dose; $n=10$ ), respectively. Diabetic rats that received nimodipine prior to ischemia-reperfusion injury were the positive control group (Nimo group; $\mathrm{n}=10 ; 5 \mathrm{mg} / \mathrm{kg} / \mathrm{day}$; intragastrically). All groups were administered with the corresponding agents for three weeks before induction of ischemia. All rats were anesthetized with pentobarbital $(45 \mathrm{mg} / \mathrm{kg}$; intraperitoneal injection) and sacrificed using cervical dislocation. Rat brains and blood samples were collected. The experimental protocol is presented in Fig. 1.

Neurological deficit evaluation. Neurological function was evaluated 2 and $24 \mathrm{~h}$ after reperfusion by an investigator blinded to the study groups: 0 , no deficit; 1 , failure to extend right forelimb while the tail was pulled; 2 , spontaneous circling or walking to the contralateral side; 3 , stumble only when stimulated with a depressed level of consciousness; 4 , unresponsive to stimulation.

Infarct volume measurement. 2,3,5-Triphenyltetrazolium chloride (TTC) staining was performed according to previous descriptions (36) for the evaluation of the infarct volume in experimental ischemic stroke. TTC stained the normal cerebral areas deep red without any effect on the infarct tissue, which enables identification of the healthy regions from the infarcted areas. A total of 5 rats from each group were used for infarct volume measurement ( $n=5$ per group). Rats were euthanized and brains were removed immediately. Brain samples were placed in a brain matrix and sliced into $2 \mathrm{~mm}$ sections. The slices were incubated in a $2 \%$ solution of TTC (cat. no. 129K1867V; Sigma-Aldrich; Merck KGaA) at $37^{\circ} \mathrm{C}$ for $30 \mathrm{~min}$, then fixed in a $4 \%$ buffered paraformaldehyde solution and scanned using a scanner (EPSON Perfection V33). The infarct area and the hemisphere area of each section were traced and quantified using ImageJ software (National Institutes of Health) and expressed as the percent of infarct area in the whole brain.

S100B measurement. The amount of serum S100B protein was detected using a commercially available ELISA kit (cat. no. 1302271; Shanghai Bio-Tech Co., Ltd.), according to the manufacturer's protocol, and expressed as $\mathrm{ng} / \mathrm{ml}$.

Measurement of malondialdehyde level. The right striatum $(50 \mathrm{mg})$ was homogenized with $450 \mu \mathrm{l} 0.9 \% \mathrm{NaCl}$ and centrifuged at $4^{\circ} \mathrm{C}$ at $12,000 \mathrm{x}$ g for $15 \mathrm{~min}$. A total of $20 \mu \mathrm{l} 6 \mathrm{~mol} / \mathrm{l}$
$\mathrm{NaOH}$ was then added to $100 \mu \mathrm{l}$ supernatant in an Eppendorf tube and the sample was incubated in a water bath at $60^{\circ} \mathrm{C}$ for $30 \mathrm{~min}$. The hydrolyzed sample was acidified with $50 \mu 135 \%$ $(v / v)$ perchloric acid. The resulting suspension was then mixed on a vortex for $30 \mathrm{sec}$ and centrifuged at $12,000 \mathrm{x} \mathrm{g}$ for $10 \mathrm{~min}$. A total of $200 \mu \mathrm{l}$ top clear supernatant was transferred to a $1.5 \mathrm{ml}$ Eppendorf tube. The resultant supernatant was mixed with $20 \mu 1$ 2,4-dinitrophenylhydrazine solution $(5 \mathrm{mmol} / \mathrm{l}$ in $2 \mathrm{~mol} / \mathrm{l} \mathrm{HCl} ; \mathrm{pH}=0.09$ ) and incubated at room temperature for $30 \mathrm{~min}$. After derivatization, samples were filtered through a $0.22 \mu \mathrm{m}$ filter. Aliquots of $50 \mu \mathrm{l}$ were injected into a HPLC system (37), in which an Agilent Zorbax SB-C18 column (250x4.6 mm; Agilent Technologies, Inc.) was used. The mobile phase was acetonitrile-distilled water $(38: 62, v / v)$ containing $0.2 \%(v / v)$ acetic acid at a flow rate of $1.0 \mathrm{ml} / \mathrm{min}$, and the wavelength of the UV detector was set at $310 \mathrm{~nm}$. The level of striatum malondialdehyde (MDA) was expressed as $\mu \mathrm{mol} / \mathrm{g}$ protein, and protein concentration was determined using a bicinchoninic acid (BCA) assay.

Measurement of glutathione (GSH) content. The level of GSH was measured as previously described by Liu et al (38). The compound 3-carboxy-4-nitrophenyl disulfide can react with sulfhydryl compounds (including GSH) and form a yellow compound with a strong absorption at $420 \mathrm{~nm}$. The measurement of GSH was performed using a commercial kit (cat. no. A006-1; Nanjing Jiancheng Bioengineering Institute), and the level of striatum GSH was expressed as $\mathrm{mg} / \mathrm{g}$ protein.

Superoxide dismutase (SOD) activity assay. SOD is an important antioxidative enzyme, and, in the current study, its activity was determined according to the method of Sun et al (39). This method uses the inhibition of nitroblue tetrazolium reduction by the xanthine and xanthine oxidase system as a superoxide generator. SOD activity was subsequently measured at $550 \mathrm{~nm}$ by the degree of inhibition using a commercial kit (cat. no. A001-1; Nanjing Jiancheng Bioengineering Institute). A total of one unit of enzyme was defined as the amount of enzyme required at an inhibitory rate of $50 \%$. The activity of SOD was expressed as units/mg protein.

Western blot analysis. A total of 5 rats from each group were used for western blot analysis ( $\mathrm{n}=5$ per group). After weighing the rats, the right hippocampus were dissected and homogenized using a sonicator with six-fold volumes $(w / v)$ of $50 \mathrm{mmol} / \mathrm{l}$ Tris buffer (pH=7.4) containing $150 \mathrm{mmol} / \mathrm{l} \mathrm{NaCl}$, $1 \mathrm{mmol} / \mathrm{l} \mathrm{EDTA}, 1 \mathrm{mmol} / \mathrm{l} \mathrm{PMSF}, 1 \mathrm{mmol} / 1 \mathrm{Na}_{3} \mathrm{VO}_{4}, 2 \mathrm{mmol} / 1$ 
DTT and $50 \mathrm{mmol} / \mathrm{l} \mathrm{NaF}$, in an ice-cold bath. Samples were left at $4^{\circ} \mathrm{C}$ for at least $30 \mathrm{~min}$, the homogenates were then centrifuged at $4^{\circ} \mathrm{C}$ at $10,000 \mathrm{x}$ g for $15 \mathrm{~min}$ and the supernatant was collected and denatured in SDS. The protein concentration in the supernatant was determined using a BCA protein assay kit (Thermo Fisher Scientific, Inc.).

The same amount of protein $(80 \mu \mathrm{g})$ was electrophoresed on $8 \%$ SDS-PAGE and transferred to a PVDF membrane. The membranes were incubated overnight at $4^{\circ} \mathrm{C}$ with primary antibodies, including Akt Rabbit monoclonal antibody (1:1,000; cat. no. 4691; Cell Signaling Technology, Inc.), p-Akt (Ser473) Rabbit monoclonal antibody (1:1,000; cat. no. 4060; Cell Signaling Technology, Inc.), mTOR Rabbit monoclonal antibody (1:1,000; cat. no. 2983; Cell Signaling Technology, Inc.), p-mTOR (Ser2448) Rabbit monoclonal antibody (1:1,000; cat. no. 5536; Cell Signaling Technology, Inc.), GLT1 Rabbit polyclonal antibody (1:1,000; cat. no. ab106289; Abcam), and $\beta$-actin Rabbit polyclonal antibody (1:1,000; cat. no. AP0060; Bioworld Technology, Inc.), respectively. The membranes were washed and incubated with alkaline phosphatase-conjugated IgG (1:10,000; cat. no. E030220-02; EarthOx Life Sciences) at room temperature for $2 \mathrm{~h}$ before being exposed to BCIP/NBT alkaline phosphatase color developing reagent (cat. no. C3206; Beyotime Institute of Biotechnology) for $15 \mathrm{~min}$. Western blot density was measured using Image J software (Rawak Software, Inc.) and normalized using $\beta$-actin as an internal control.

Statistical analysis. All data in the different experimental groups were expressed as the mean $\pm \mathrm{SD}$. Data were analyzed using GraphPad Prism (version 5.0; GraphPad Software, Inc.). A comparison between groups was conducted using one-way ANOVA, followed Tukey's multiple comparisons tests. $\mathrm{P}<0.05$ and $\mathrm{P}<0.01$ were considered to indicate a statistically significant difference.

\section{Results}

Effect of GbE on body weights and fasting blood glucose. The body weights of rats were measured after STZ injection (day 0 ) and on days 7, 14, 21 and 28. As presented in Fig. 2, the original body weights of eight groups were $\sim 200 \mathrm{~g}$. After STZ injection, the body weights of diabetic rats (STZ + sham group and STZ + I/R group) remained unchanged, whereas a marked increase was observed in nondiabetic rats (Con + sham group and Con $+\mathrm{I} / \mathrm{R}$ group) during the 4 consecutive weeks $(\mathrm{P}<0.01) . G b \mathrm{E}(50,100$ and $200 \mathrm{mg} / \mathrm{kg} /$ day $)$ was administered intragastrically once per day from day 7 to day 28 , and there were no significant differences among the groups at each time point.

Fasting blood glucose levels were measured on days 7 (after a week of STZ injection) and 28 (after 3 weeks of consecutive administration). On days 7 and 28 , the fasting blood glucose levels in diabetic rats was significantly increased compared with nondiabetic rats $(\mathrm{P}<0.01$; Table I). No significant differences in fasting blood glucose levels were observed in the three $G b \mathrm{E}$ groups and the Nimo group.

Effect of GbE on neurological deficits. Neurological deficits were evaluated 2 and $24 \mathrm{~h}$ after reperfusion. Compared with the $2 \mathrm{~h}$ time point after reperfusion, $\mathrm{I} / \mathrm{R}$ injury in nondiabetic
Table I. Effect of $G b \mathrm{E}$ on the fasting blood glucose of STZ-induced diabetic rats subjected to $30 \mathrm{~min} \mathrm{MCAO} / 24 \mathrm{~h}$ reperfusion.

\begin{tabular}{lcr}
\hline & \multicolumn{2}{c}{ Fasting blood glucose $(\mathrm{mmol} / \mathrm{l})$} \\
\cline { 2 - 3 } Groups & \multicolumn{1}{c}{ Day 7} & Days 28 \\
\hline Con + sham & $5.75 \pm 1.28$ & $4.99 \pm 0.49$ \\
Con + I/R & $5.89 \pm 1.48$ & $5.16 \pm 0.59$ \\
STZ + sham & $22.38 \pm 2.21^{\mathrm{a}}$ & $26.90 \pm 3.44^{\mathrm{a}}$ \\
STZ + I/R & $22.51 \pm 4.32^{\mathrm{b}}$ & $27.63 \pm 3.46^{\mathrm{b}}$ \\
Nimo & $19.42 \pm 1.87$ & $24.44 \pm 6.81$ \\
GL & $21.03 \pm 4.42$ & $26.64 \pm 3.90$ \\
GM & $21.22 \pm 4.74$ & $27.85 \pm 5.39$ \\
GH & $21.33 \pm 5.41$ & $24.41 \pm 5.42$ \\
\hline
\end{tabular}

Data are expressed as mean $\pm \mathrm{SD}(\mathrm{n}=10$ per time point $) .{ }^{\mathrm{a}} \mathrm{P}<0.01 \mathrm{vs}$. Con + sham group; ${ }^{b} \mathrm{P}<0.01$ vs. Con $+\mathrm{I} / \mathrm{R}$ group. GbE, Ginkgo biloba extract; STZ, streptozotocin; MCAO, middle cerebral artery occlusion; Con, control; I/R, ischemia-reperfusion; Nimo, nimodipine group; GL, $G b \mathrm{E}$ low dose group; GM, $G b \mathrm{E}$ moderate dose group; $\mathrm{GH}, G b \mathrm{E}$ high dose group.

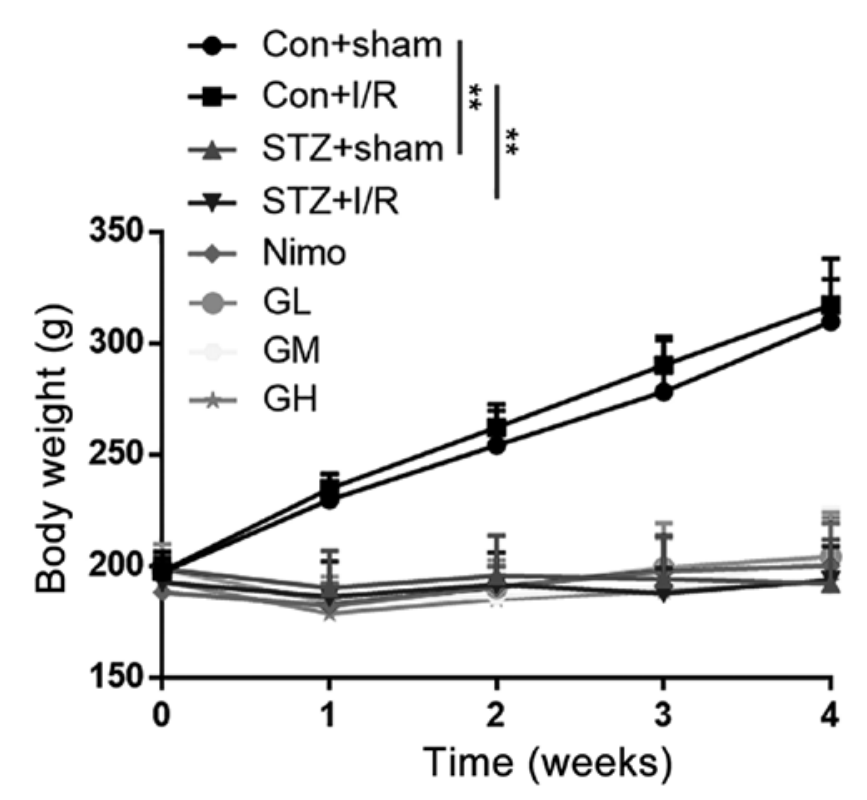

Figure 2. Effect of $G b \mathrm{E}$ on the body weight of STZ-induced diabetic rats subjected to $30 \mathrm{~min} \mathrm{MCAO} / 24 \mathrm{~h}$ reperfusion. Data are expressed as mean \pm standard deviation ( $\mathrm{n}=10$ per time point). ${ }^{* *} \mathrm{P}<0.01 . G b \mathrm{E}$, Ginkgo biloba extract; STZ, streptozotocin; MCAO, middle cerebral artery occlusion; Con, control; I/R, ischemia-reperfusion; Nimo, nimodipine group; $\mathrm{GL}, G b \mathrm{E}$ low dose group; GM, $G b \mathrm{E}$ moderate dose group; $\mathrm{GH}, G b \mathrm{E}$ high dose group.

rats (Con $+\mathrm{I} / \mathrm{R}$ group) had severe to mild neurological deficits $(\mathrm{P}<0.01)$, whereas I/R injury in diabetic rats $(\mathrm{STZ}+\mathrm{I} / \mathrm{R}$ group) resulted in severe to very severe neurological deficits $24 \mathrm{~h}$ after reperfusion $(\mathrm{P}<0.01)$, and sham-operated animals did not exhibit any deficits (Table II). The Nimo and three $G b \mathrm{E}$ dose groups had significantly improved neurological scores at $24 \mathrm{~h}$ of reperfusion $(\mathrm{P}<0.01$; Table II). 
Table II. Effect of $G b \mathrm{E}$ on the behavioral scores of neurological function of rats 2 and $24 \mathrm{~h}$ after reperfusion.

Neurological scores (mean \pm SD)

\begin{tabular}{lcccc} 
& 2 h after reperfusion & 24 h after reperfusion & $n$ & Neurological deficits \\
\hline Con + sham & 0 & 0 & 10 & None \\
Con + I/R & $1.92 \pm 0.57$ & $0.30 \pm 0.67^{\mathrm{a}}$ & 10 & Severe $\rightarrow$ mild \\
STZ + sham & 0 & 0 & 10 & None \\
STZ+I/R & $2.00 \pm 0.67$ & $2.90 \pm 0.57^{\mathrm{a}}$ & 10 & Severe $\rightarrow$ very severe \\
Nimo & $2.10 \pm 0.57$ & $1.00 \pm 0.47^{\mathrm{a}}$ & 10 & Severe $\rightarrow$ mild \\
GL & $2.00 \pm 0.47$ & $1.10 \pm 0.57^{\mathrm{a}}$ & 10 & Severe $\rightarrow$ mild \\
GM & $2.10 \pm 0.74$ & $0.80 \pm 0.63^{\mathrm{a}}$ & 10 & Severe $\rightarrow$ mild \\
GH & $2.00 \pm 0.67$ & $0.70 \pm 0.48^{\mathrm{a}}$ & 10 & Severe $\rightarrow$ mild
\end{tabular}

Data are expressed as mean \pm standard deviation ( $\mathrm{n}=10$ for each group). ${ }^{\mathrm{a}} \mathrm{P}<0.01 \mathrm{vs} .2 \mathrm{~h}$ time point after reperfusion of each group. GbE, Ginkgo biloba extract; STZ, streptozotocin; MCAO, middle cerebral artery occlusion; Con, control; I/R, ischemia-reperfusion; Nimo, nimodipine group; GL, GbE low dose group; GM, GbE moderate dose group; GH, GbE high dose group.
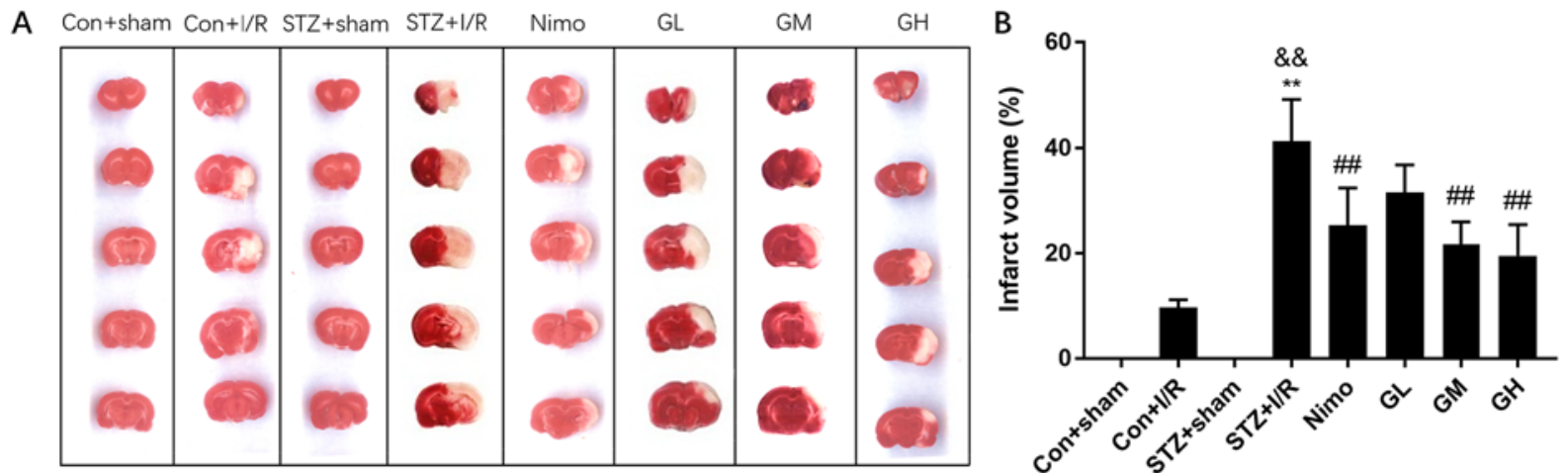

Figure 3. Effect of $G b \mathrm{E}$ on the infarct volume of STZ-induced diabetic rats subjected to $30 \mathrm{~min} \mathrm{MCAO} / 24 \mathrm{~h}$ reperfusion. TTC stained brain slices of rats in different groups. (A) The normal brain areas are red and the infarct areas are white. (B) Infarct volume of rats in different groups. Data are expressed as mean \pm standard deviation $(\mathrm{n}=5) .{ }^{* *} \mathrm{P}<0.01$ vs. $\mathrm{STZ}+$ sham group; ${ }^{\&}{ }^{\&} \mathrm{P}<0.01$ vs. Con $+\mathrm{I} / \mathrm{R}$ group; ${ }^{\# \#} \mathrm{P}<0.01$ vs. STZ + I/R group. GbE, Ginkgo biloba extract; STZ, streptozotocin; MCAO, middle cerebral artery occlusion; TTC, 2,3,5-Triphenyltetrazolium chloride; Con, control; I/R, ischemia-reperfusion; Nimo, nimodipine group; GL, $G b \mathrm{E}$ low dose group; GM, $G b \mathrm{E}$ moderate dose group; $\mathrm{GH}, G b \mathrm{E}$ high dose group.

Effect of GbE on cerebral infarct volume. The effects of GbE on rat infarct volume were investigated using TTC staining. No lesion was observed in sham-operated groups. Con $+\mathrm{I} / \mathrm{R}$ rats that were subjected to 30 min MCAO followed by $24 \mathrm{~h}$ reperfusion presented smaller infarct volumes of $9.80 \pm 1.48 \%$. The STZ + I/R group exhibited markedly increased infarct volume percentages $(41.34 \pm 7.88 \%)$ compared with the Con + I/R group ( $\mathrm{P}<0.01 ;$ Fig. 3B). Intermediate and high doses of $G b \mathrm{E}$, and nimodipine markedly reduced the infarct volume ( $\mathrm{P}<0.01$; Fig. 3B). However, the low dose of $G b \mathrm{E}$ had little effect on it.

Effect of GbE on S100B in serum of rats. S100 calcium-binding protein B (S100B), which is a biomarker of traumatic brain injury, is primarily expressed in the central nervous system by astrocytes (40). Ischemia is associated with the increased expression of S100B, which may be released from damaged astrocytes (41). The level of serum S100B is an indicator of brain injury following stroke (42). Therefore, the concentration of S100B was examined using an ELISA to investigate the neuroprotective effect of $G b \mathrm{E}$. As presented in Fig. 4,

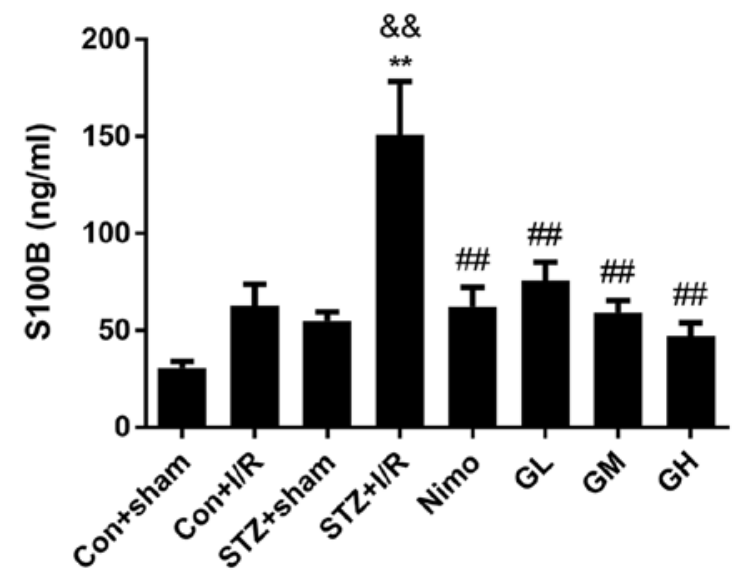

Figure 4. Effect of $G b \mathrm{E}$ on the content of serum S100B in diabetic rats subjected to $30 \mathrm{~min} \mathrm{MCAO} / 24 \mathrm{~h}$ reperfusion. Data are expressed as mean \pm standard deviation $(\mathrm{n}=10) .{ }^{* *} \mathrm{P}<0.01$ vs. $\mathrm{STZ}+$ sham group, ${ }^{\& \&} \mathrm{P}<0.01$ vs. Con + I/R group, ${ }^{\# \#} \mathrm{P}<0.01$ vs. $\mathrm{STZ}+\mathrm{I} / \mathrm{R}$ group. GbE, Ginkgo biloba extract; STZ, streptozotocin; MCAO, middle cerebral artery occlusion; Con, control; I/R, ischemia-reperfusion; Nimo, nimodipine group; GL, $G b \mathrm{E}$ low dose group; GM, GbE moderate dose group; $\mathrm{GH}, G b \mathrm{E}$ high dose group. 

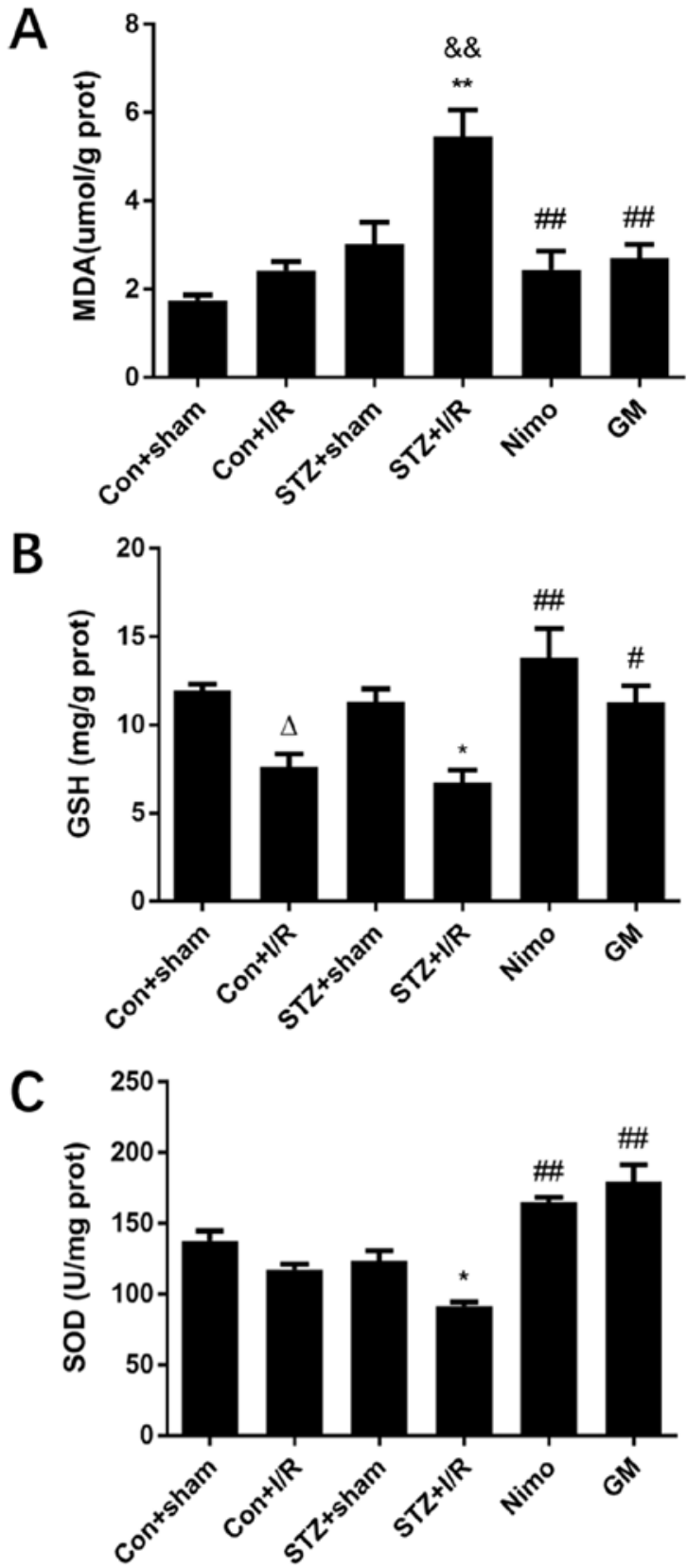

Figure 5. Effect of $G b \mathrm{E}$ on oxidative stress in the striatums of rats. (A) MDA, (B) GSH and (C) SOD were determined. Data are expressed as mean \pm standard deviation $(\mathrm{n}=5) .{ }^{\Delta} \mathrm{P}<0.05$ vs. Con + sham group; ${ }^{*} \mathrm{P}<0.05$ or ${ }^{* *} \mathrm{P}<0.01$ vs. STZ + sham group; ${ }^{\& \&} \mathrm{P}<0.01$ vs. Con $+\mathrm{I} / \mathrm{R}$ group; ${ }^{\#} \mathrm{P}<0.05$ or ${ }^{\# \#} \mathrm{P}<0.01$ vs. STZ + I/R group. GbE, Ginkgo biloba extract; MDA, malondialdehyde GSH, glutathione; SOD, superoxide dismutase; STZ, streptozotocin; Con, control; I/R, ischemia-reperfusion; Nimo, nimodipine group; GM, GbE moderate dose group.

in diabetic rats, $\mathrm{I} / \mathrm{R}$ injury significantly increased the level of serum $\mathrm{S} 100 \mathrm{~B}(\mathrm{P}<0.01)$. STZ-induced diabetic increased $\mathrm{S} 100 \mathrm{~B}$ level $(\mathrm{P}<0.01)$ in $\mathrm{STZ}+\mathrm{I} / \mathrm{R}$ group compared with the nondiabetic rats with I/R injury (Con $+\mathrm{I} / \mathrm{R}$ group). However, pretreatment with nimodipine and $G b \mathrm{E}$ significantly decreased $\mathrm{S} 100 \mathrm{~B}$ compared with the $\mathrm{STZ}+\mathrm{I} / \mathrm{R}$ group $(\mathrm{P}<0.01)$.

Increasing doses of $G b \mathrm{E}$ resulted in a stepped decrease of infarct volume and S100B level. The moderate dose of $G b \mathrm{E}$ $(100 \mathrm{mg} / \mathrm{kg}, \mathrm{GM})$ was subsequently used for the following experiments.
Effect of GbE on oxidative stress in the rat striatum. One potential mechanism for diabetes and its complications is oxidative stress (43). To investigate whether the neuroprotective effect of $G b \mathrm{E}$ was associated with the decrease in oxidative stress levels, three associated molecules were examined, including MDA, GSH and SOD. As presented in Fig. 5, in nondiabetic rats, compared with the Con + sham group, I/R injury significantly decreased GSH level $(\mathrm{P}<0.05)$. In diabetic rats, I/R injury significantly caused oxidative stress damage, and this was indicated by increased MDA level $(\mathrm{P}<0.01)$, and decreased GSH $(\mathrm{P}<0.05)$ and decreased SOD activity $(\mathrm{P}<0.05)$. Compared with nondiabetic rats with $\mathrm{I} / \mathrm{R}$ injury (Con + I/R group), STZ-induced diabetic rats exhibited increased MDA level $(\mathrm{P}<0.01)$. However, $G b \mathrm{E}$ pretreatment significantly suppressed MDA level $(\mathrm{P}<0.01)$, and inhibited the decrease of GSH $(\mathrm{P}<0.05)$ and SOD $(\mathrm{P}<0.01)$.

Effect of GbE on the quantities of p-Akt and p-mTOR in the hippocampus of rats. It was previously revealed that $G b \mathrm{E}$ pretreatment improved neurological deficits, reduced the infarct volume and relieved oxidative stress following cerebral $\mathrm{I} / \mathrm{R}$ injury in diabetic rats. To elucidate the mechanism by which $G b \mathrm{E}$ ameliorated neuronal damage, western blot analysis was used to identify associated protein expression. The results indicated that in diabetic rats, I/R injury decreased the expression of p-Akt ( $\mathrm{P}<0.01$; Fig. 6A). Compared with nondiabetic rats with I/R injury (Con + I/R group), STZ-induced diabetic decreased p-Akt/Akt ratio $(\mathrm{P}<0.01)$ in the $\mathrm{STZ}+\mathrm{I} / \mathrm{R}$ group. However, in the nimodipine and $G b$ E-pretreated groups, the ratio of $\mathrm{p}$-Akt/Akt was significantly increased compared with the STZ + I/R group $(\mathrm{P}<0.01$; Fig. $6 \mathrm{~A})$. Variation in the $\mathrm{p}-\mathrm{mTOR} / \mathrm{mTOR}$ ratio was consistent with the change in the p-Akt/Akt ratio (Fig. 6B).

GbE inhibits ischemia-induced downregulation of GLT1 in diabetic rats subjected to $30 \mathrm{~min} M C A O / 24 \mathrm{~h}$ reperfusion. A recent study has revealed that glutamate uptake exhibits a protective function in hippocampal astrocytes (44). Therefore, in the current study, the hippocampus was collected to elucidate the possible mechanism underlying the neuroprotective effect of $G b \mathrm{E}$ against injury. The results indicated that whether in nondiabetic or diabetic rats, I/R injury decreased the expression of GLT1 ( $\mathrm{P}<0.01$ or $\mathrm{P}<0.05$; Fig. $6 \mathrm{C})$. Compared with nondiabetic rats with $\mathrm{I} / \mathrm{R}$ injury (Con $+\mathrm{I} / \mathrm{R}$ group), STZ-induced diabetic rats exhibited decreased GLT1 expression $(\mathrm{P}<0.01)$ in the $\mathrm{STZ}+\mathrm{I} / \mathrm{R}$ group. However, in the nimodipine and $G b \mathrm{E}$-pretreated groups, the expression of GLT1 was significantly increased compared with the STZ + I/R group ( $\mathrm{P}<0.01$; Fig. 6C).

\section{Discussion}

Ischemic stroke generally occurs in diabetic patients with poor glycemic control (1). It has been well established that patients with hyperglycemia are four to five times more likely to suffer from a stroke compared with patients with normoglycemia, with worse neurologic outcomes $(5,45)$. The present work demonstrated in vivo evidence of the protective effect of $G b \mathrm{E}$ against cerebral ischemic injury in diabetic rats. These results indicated that cerebral injury of $30 \mathrm{~min} \mathrm{MCAO} / 24 \mathrm{~h}$ 
A
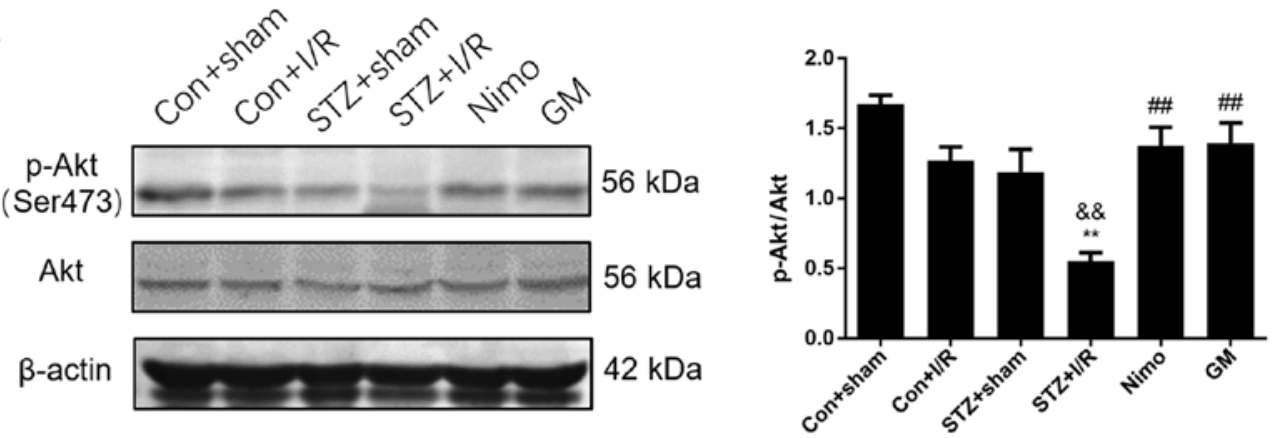

B
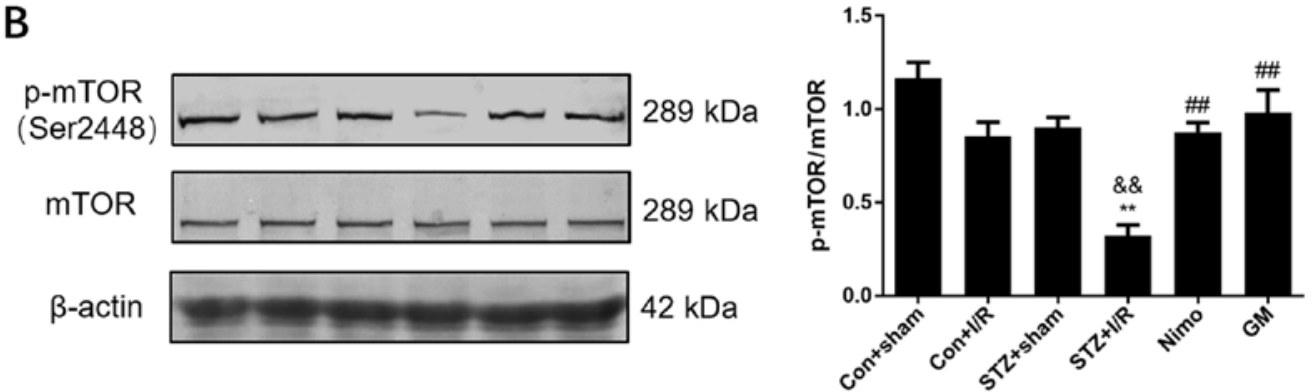

C
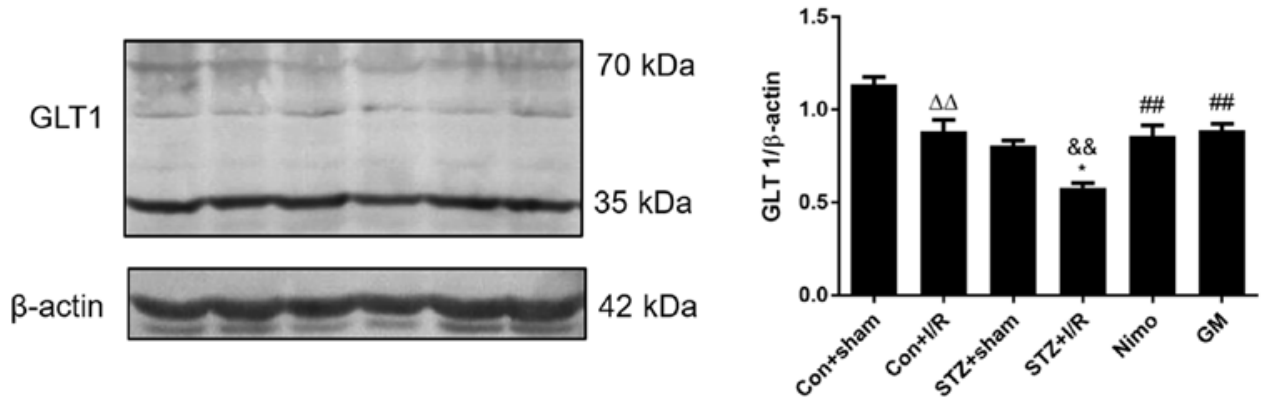

Figure 6. Effect of $G b \mathrm{E}$ on the protein expression of (A) p-Akt, (B) p-mTOR and (C) GLT1 in the hippocampus of rats. Data are expressed as mean \pm standard deviation $(\mathrm{n}=5) .{ }^{\Delta} \mathrm{P}<0.01$ vs. Con + sham group; ${ }^{*} \mathrm{P}<0.05$ or ${ }^{* *} \mathrm{P}<0.01 \mathrm{vs}$. STZ + sham group; ${ }^{\&} \mathrm{P}<0.01 \mathrm{vs}$. Con $+\mathrm{I} / \mathrm{R}$ group; ${ }^{\#} \mathrm{P}<0.01$ vs. STZ+I/R group. GbE, Ginkgo biloba extract; GLT1, glutamate transporter 1; STZ, streptozotocin; Con, control; I/R, ischemia-reperfusion; Nimo, nimodipine group; GM, GbE moderate dose group.

reperfusion in STZ induced diabetic rats causes more damage compared with nondiabetic rats. However, pretreatment with $G b \mathrm{E}$ protected against cerebral I/R injury in diabetic rats, which may be associated with the inhibition of oxidative stress, the activation of Akt/mTOR signaling cascade and the upregulation of GLT1 expression.

The current study indicated that a single injection of STZ significantly prevented weight gain and increased fasting blood glucose level compared with normal control rats. However, $G b \mathrm{E}$ exhibited no effect on body weight or fasting blood glucose level. Furthermore, it was demonstrated that $G b \mathrm{E}$ exhibited a protective effect on cerebral ischemic injury in diabetic rats, which was assessed by measuring the neurological scores, infarct volume and serum S100B level. The current study revealed that whether in nondiabetic or diabetic rats, deficits in performance due to I/R injury, significantly reduced the neurological deficit following $2 \mathrm{~h}$ of reperfusion. However, long term reperfusion ameliorated injury of nondiabetic rats and aggravated diabetic rats, which may be due to high glucose levels preventing the repair of ischemic penumbra and resultant brain tissue damage (46). GbE pretreatment exhibited a markedly decreased neurological deficit. The cerebral infarct volume of $24 \mathrm{~h}$ following reperfusion was consistent with the results of neurological deficits. Furthermore, it was revealed that the dose-dependent administration of $G b \mathrm{E}$ significantly decreased serum S100B level. The mechanism by which $G b \mathrm{E}$ protects against cerebral ischemic injury in diabetic rats was subsequently assessed.

A large number of ROS are produced during cerebral ischemia and excessively consume the endogenous antioxidative proteins, leading to the changes in the expression and activity of SOD and GSH (47). The decreased activity of antioxidant enzymes reduces the ability of brain tissue to scavenge ROS (48). MDA is the final product of lipid peroxidation and indirectly reflects changes in ROS content (49). The results of the current study indicated that $G b \mathrm{E}$ enhanced antioxidant enzyme activities and reduced lipid peroxidation.

The hippocampus is an essential brain region which plays roles in memory forming, organizing, and storing. Brian ischemia leads to movement, visual, sensory, and behavioral disorders, especially aphasia and impaired spatial learning $(50,51)$. Neurons in the hippocampal CA1 regions are very sensitive, and they quickly react to brain I/R $(52,53)$. Studies have demonstrated that hippocampal areas of the mouse brain are more vulnerable to neuron death following an ischemic insult (54). Several researchers 
chose the hippocampus to study Akt pathway in rat models of ischemic brain damage (54-56). The phosphatidylinositol-3 (PI3) kinase/Akt signal pathway enhances cell survival, proliferation and differentiation (57). It has been demonstrated that ROS regulates the PI3K/Akt pathway, which leads to changes in a number of downstream signaling proteins and induces a variety of pathophysiological responses $(58,59)$. A study performed by Wang et al (60) demonstrated that ROS overproduction, which was induced by high glucose in astrocytes, can lead to decreased cell viability and apoptosis. A number of studies have indicated that oxidative stress-induced neuronal damage and death following cerebral ischemia can be inhibited by activation of the PI3K/Akt/mTOR signaling pathway (16). In the present study, the results demonstrated that I/R injury significantly decreased the ratio of $\mathrm{p}-\mathrm{Akt} / \mathrm{Akt}$ and $\mathrm{p}-\mathrm{mTOR} / \mathrm{mTOR}$, and this was exacerbated by diabetes. However, $G b \mathrm{E}$ pretreatment markedly reversed this decreased expression by upregulating the ratio of p-Akt/Akt and $\mathrm{p}-\mathrm{mTOR} / \mathrm{mTOR}$. This result suggested that $G b \mathrm{E}$, which is a free radical scavenger, could attenuate I/R injury in diabetic rats by activating the $\mathrm{Akt} / \mathrm{mTOR}$ signal cascade.

Glutamate is the most abundant neurotransmitter in the cerebral neural system (61). GLT1, which is also known as EAAT2, is the predominant subtype that performs the majority of glutamate reuptake (19). Extracellular glutamate concentration is mediated primarily by the astrocytic glutamate transporter GLT1. However, disruption of glutamate transporter activity and expression can lead to excitotoxicity and is implicated in ischemic events $(20,21)$. It has been demonstrated that Akt is a key regulator of GLT1 expression (23). Additionally, mTOR, which is a downstream target of the PI3K/Akt pathway, is associated with the regulation of GLT1 (22). Previous reports have demonstrated that GLT1 knock-down exacerbates neuronal damage in ischemia rats (21), whereas GLT1 upregulation reduces cerebral ischemic injury in hyperglycemic rats (34). In the current study, I/R injury was demonstrated to downregulate GLT1 expression in diabetic rats, and $G b \mathrm{E}$ resulted in the upregulation of GLT1 expression, suggesting that $G b \mathrm{E}$ might serve an important role in protecting against I/R injury in diabetic rats via resistance to glutamate excitotoxicity. The results of the present study are supported by a study performed by Mdzinarishvili et al (62) that demonstrated that EGb761 reduces the release of glutamate in the brain of ischemic mice by monitoring extracellular glutamate concentration, however the underlying mechanism for this was not determined.

Previous studies have identified that the neuron-dependent regulation of GLT 1 transcription requires NF- $\kappa B$ and $\mathrm{\kappa B}$ motif-binding phosphoprotein $(23,63)$. Therefore, the downstream transcription factor(s) require identification in future studies. Additionally, nimodipine, which is a positive control drug used in the present study, is a $\mathrm{Ca}^{2+}$ channel blocker. The protective effect of nimodipine is positive, which indicated that the experimental design of the current study is feasible. Nimodipine worked as well as $G b \mathrm{E}$ in the present study, however nimodipine can lead to hypotension (64), while the incidence of adverse reactions by $G b \mathrm{E}$ is low. Additionally, $G b \mathrm{E}$ may also serve a role in lowering blood glucose (65). As an effective adjuvant drug, $G b$ E exhibits a glycemic control value in the clinical treatment of patients with T2DM (66).
In conclusion, the results of the current study demonstrated that $G b \mathrm{E}$ pretreatment ameliorated neurological deficit, reduced infarct volume, decreased S100B level, inhibited oxidative stress, and upregulated the expression of Akt/mTOR and GLT1 in diabetic rats with cerebral ischemia-reperfusion injury. However, further studies using cell models are required to determine the in vitro protective effect and to clarify the accurate mechanism of the effect of $G b \mathrm{E}$ in cerebral ischemia-reperfusion injury.

\section{Acknowledgements}

Not applicable.

\section{Funding}

The current study was supported by the Open Foundation (grant no. KJS1107) from the Key Laboratory of Anesthesiology and Discipline Construction Projects of Jiangsu Province, the College Graduate Research and Innovation Projects in Jiangsu Province (grant no. CXZZ_1000).

\section{Availability of data and materials}

The datasets used and/or analyzed during the current study are available from the corresponding author on reasonable request.

\section{Authors' contributions}

ML and SG designed the study. MY, ML, ZS, TM and XM performed the experiments. MY and ML collected and analyzed the experimental data. MY drafted the manuscript. All authors read and approved the final manuscript.

\section{Ethics approval and patient consent to participate}

All experimental and surgical procedures for animals were strictly performed in accordance with the Guiding Principles for Care and Use of Laboratory Animals of Xuzhou Medical University. The present study was approved by the Ethics Committee of Xuzhou Medical University.

\section{Patient consent for publication}

Not applicable.

\section{Competing interests}

The authors declare that they have no competing interests.

\section{References}

1. Luitse MJ, Biessels GJ, Rutten GE and Kappelle LJ: Diabetes, hyperglycaemia, and acute ischaemic stroke. Lancet Neurol 11: 261-271, 2012.

2. Barrett-Connor E and Khaw KT: Diabetes mellitus: An independent risk factor for stroke? Am J Epidemiol 128: 116-123, 1988.

3. Muranyi M, Fujioka M, He Q, Han A, Yong G, Csiszar K and Li PA: Diabetes activates cell death pathway after transient focal cerebral ischemia. Diabetes 52: 481-486, 2003.

4. Rizk NN, Rafols J and Dunbar JC: Cerebral ischemia induced apoptosis and necrosis in normal and diabetic rats. Brain Res 1053: 1-9, 2005. 
5. Els T, Klisch J, Orszagh M, Hetzel A, Schulte-Mönting J, Schumacher M and Lucking CH: Hyperglycemia in patients with focal cerebral ischemia after intravenous thrombolysis: Influence on clinical outcome and infarct size. Cerebrovasc Dis 13: 89-94, 2002.

6. Emerging Risk Factors Collaboration; Sarwar N, Gao P, Seshasai SR, Gobin R, Kaptoge S, Di Angelantonio E, Ingelsson E, Lawlor DA, Selvin E, et al: Diabetes mellitus, fasting blood glucose concentration, and risk of vascular disease: A collaborative meta-analysis of 102 prospective studies. Lancet 9733: 2215-2222, 2010.

7. Almdal T, Scharling H, Jensen JS and Vestergaard H: The independent effect of type 2 diabetes mellitus on ischemic heart disease, stroke and death: A population-based study of 13,000 men and women with 20 years of follow-up. Arch Intern Med 164: 1422-1426, 2004.

8. Giacco F and Brownlee M: Oxidative stress and diabetic complications. Circ Res 107: 1058-1070, 2010.

9. Brownlee M: Biochemistry and molecular cell biology of diabetic complications. Nature 6865: 813-820, 2001.

10. Adibhatla RM and Hatcher JF: Lipid oxidation and peroxidation in CNS health and disease: from molecular mechanisms to therapeutic opportunities. Antioxid Redox Signal 12: 125-169, 2010.

11. Ran Z, Zhang Y, Wen X and Ma J: Curcumin inhibits high glucose-induced inflammatory injury in human retinal pigment epithelial cells through the ROSPI3K/AKT/mTOR signaling pathway. Mol Med Rep 19: 1024-1031, 2019.

12. Pan Y, Wang N, Xia P, Wang E, Guo Q and Ye Z: Inhibition of Racl ameliorates neuronal oxidative stress damage via reducing Bcl-2/Rac1 complex formation in mitochondria through PI3K/Akt/mTOR pathway. Exp Neurol 300: 149-166, 2018.

13. Maiese K, Chong ZZ, Wang S and Shang YC: Oxidant stress and signal transduction in the nervous system with the PI3-K, Akt and mTOR cascade. Int J Mol Sci 13: 13830-13866, 2012.

14. Dong L, Zhou S, Yang X, Chen Q, He Y and Huang W: Magnolol protects against oxidative stress-mediated neural cell damage by modulating mitochondrial dysfunction and PI3K/Akt signaling. J Mol Neurosci 50: 469-481, 2013.

15. Koh PO: Melatonin prevents ischemic brain injury through activation of the mTOR/p70S6 kinase signaling pathway. Neurosci Lett 444: 74-78, 2008.

16. Yan BC, Wang J, Rui Y, Cao J, Xu P, Jiang D, Zhu X, Won MH, Bo P and Su P: Neuroprotective effects of gabapentin against cerebral ischemia reperfusion-induced neuronal autophagic injury via regulation of the PI3K/Akt/mTOR signaling pathways. J Neuropathol Exp Neurol 78: 157-171, 2019.

17. Xie R, Cheng M, Li M, Xiong X, Daadi M, Sapolsky RM and Zhao $\mathrm{H}$ : Akt isoforms differentially protect against stroke-induced neuronal injury by regulating mTOR activities. J Cereb Blood Flow Metab 33: 1875-1885, 2013.

18. Tymianski M: Emerging mechanisms of disrupted cellular signaling in brain ischemia. Nat Neurosci 14: 1369-1373, 2011.

19. Danbolt NC: Glutamate uptake. Prog Neurobiol 65: 1-105, 2001.

20. Camacho A and Massieu L: Role of glutamate transporters in the clearance and release of glutamate during ischemia and its relation to neuronal death. Arch Med Res 37: 11-18, 2006.

21. Rao VL, Dogan A, Todd KG, Bowen KK, Kim BT, Rothstein JD and Dempsey RJ: Antisense knockdown of the glial glutamate transporter GLT-1, but not the neuronal glutamate transporter EAAC1, exacerbates transient focal cerebral ischemia-induced neuronal damage in rat brain. J Neurosci 21: 1876-1883, 2001

22. $\mathrm{Wu} X$, Kihara $\mathrm{T}$, Akaike A, Niidome $\mathrm{T}$ and Sugimoto $\mathrm{H}$ : $\mathrm{PI} 3 \mathrm{~K} / \mathrm{Akt} / \mathrm{mTOR}$ signaling regulates glutamate transporter 1 in astrocytes. Biochem Biophys Res Commun 393: 514-518, 2010.

23. Ji YF, Zhou L, Xie YJ, Xu SM, Zhu J, Teng P, Shao CY, Wang Y, Luo JH and Shen Y: Upregulation of glutamate transporter GLT-1 by mTOR-Akt-NF- $\kappa \mathrm{B}$ cascade in astrocytic oxygen-glucose deprivation. Glia 61: 1959-1975, 2013.

24. Trotti D, Danbolt NC and Volterra A: Glutamate transporters are oxidant-vulnerable: A molecular link between oxidative and excitotoxic neurodegeneration? Trends Pharmacol Sci 19 328-334, 1998

25. Chen MJ, Ng JM, Peng ZF, Manikandan J, Yap YW, Llanos RM, Beart PM and Cheung NS: Gene profiling identifies commonalities in neuronal pathways in excitotoxicity: Evidence favouring cell cycle re-activation in concert with oxidative stress. Neurochem Int 62: 719-730, 2013.

26. Chan PC, Xia Q and Fu PP: Ginkgo biloba leave extract: Biological, medicinal, and toxicological effects. J Environ Sci Health C Environ Carcinog Ecotoxicol Rev 25: 211-244, 2007.
27. Ross R: Atherosclerosis-an inflammatory disease. N Engl J Med 340: 115-126, 1999

28. Springer TA: Traffic signals for lymphocyte recirculation and leukocyte emigration: The multistep paradigm. Cell 76: 301-314, 1994.

29. Akisu M, Kultursay N, Coker I and Huseyinov A: Platelet-activating factor is an important mediator in hypoxic ischemic brain injury in the newborn rat. Flunarizine and Ginkgo biloba extract reduce PAF concentration in the brain. Biol Neonate 74: 439-444, 1998.

30. Lu Q, Hao M, Wu W, Zhang N, Isaac AT, Yin J, Zhu X, Du L and Yin X: Antidiabetic cataract effects of GbE, rutin and quercetin are mediated by the inhibition of oxidative stress and polyol pathway. Acta Biochim Pol 65: 35-41, 2018.

31. Lu Q, Zuo WZ, Ji XJ, Zhou YX, Liu YQ, Yao XQ, Zhou XY, Liu YW, Zhang $F$ and Yin XX: Ethanolic Ginkgo biloba leaf extract prevents renal fibrosis through $\mathrm{Akt} / \mathrm{mTOR}$ signaling in diabetic nephropathy. Phytomedicine 22: 1071-1078, 2015.

32. Saini AS, Taliyan R and Sharma PL: Protective effect and mechanism of Ginkgo biloba extract-EGb 761 on STZ-induced diabetic cardiomyopathy in rats. Pharmacogn Mag 10: 172-178, 2014.

33. Wang J, Wang A, He H, She X, He Y, Li S, Liu L, Luo T, Huang N, Luo $\mathrm{H}$ and Zou K: Trametenolic acid B protects against cerebral ischemia and reperfusion injury through modulation of microRNA-10a and PI3K/Akt/mTOR signaling pathways. Biomed Pharmacother 112: 108692, 2019.

34. Guan T, Qian Y, Tang X, Huang M, Huang L, Li Y and Sun H: Maslinic acid, a natural inhibitor of glycogen phosphorylase, reduces cerebral ischemic injury in hyperglycemic rats by GLT-1 up-regulation. J Neurosci Res 89: 1829-1839, 2011.

35. Longa EZ, Weinstein PR, Carlson S and Cummins R: Reversible middle cerebral artery occlusion without craniectomy in rats. Stroke 20: 84-91, 1989.

36. Yang Y, Shuaib A and Li Q: Quantification of infarct size on focal cerebral ischemia model of rats using a simple and economical method. J Neurosci Methods 84: 9-16, 1998.

37. Pilz J, Meineke I and Gleiter $\mathrm{CH}$ : Measurement of free and bound malondialdehyde in plasma by high-performance liquid chromatography as the 2,4-dinitrophenylhydrazine derivative. J Chromatogr B Biomed Sci Appl 742: 315-325, 2000.

38. Liu YW, Zhu X, Li W, Lu Q, Wang JY, Wei YQ and Yin XX: Ginsenoside Re attenuates diabetes-associated cognitive deficits in rats. Pharmacol Biochem Behav 101: 93-98, 2012.

39. Sun Y, Oberley LW and Li Y: A simple method for clinical assay of superoxide dismutase. Clin Chem 34: 497-500, 1988.

40. Metting Z, Wilczak N, Rodiger LA, Schaaf JM and van der Naalt J: GFAP and S100B in the acute phase of mild traumatic brain injury. Neurology 78: 1428-1433, 2012.

41. Rothermundt M, Peters M, Prehn JH and Arolt V: S100B in brain damage and neurodegeneration. Microsc Res Tech 60: 614-632, 2003.

42. Foerch C, Wunderlich MT, Dvorak F, Humpich M, Kahles T, Goertler M, Alvarez-Sabin J, Wallesch CW, Molina CA, Steinmetz H, et al: Elevated serum S100B levels indicate a higher risk of hemorrhagic transformation after thrombolytic therapy in acute stroke. Stroke 38: 2491-2495, 2007.

43. Stadler K: Oxidative stress in diabetes. Adv Exp Med Biol 2012: 272-287, 2012.

44. Ouyang YB, Voloboueva LA, Xu LJ and Giffard RG: Selective dysfunction of hippocampal CA1 astrocytes contributes to delayed neuronal damage after transient forebrain ischemia. J Neurosci 27: 4253-4260, 2007.

45. Kaarisalo MM, Räihä I, Sivenius J, Immonen-Räihä $P$, Lehtonen A, Sarti C, Mähönen M, Torppa J, Tuomilehto J and Salomaa V: Diabetes worsens the outcome of acute ischemic stroke. Diabetes Res Clin Pract 69: 293-298, 2005.

46. Kidwell CS, Alger JR and Saver JL: Evolving paradigms in neuroimaging of the ischemic penumbra. Stroke 35 (11 Suppl 1): S2662-S2665, 2004

47. Tanaka N, Ikeda Y, Ohta Y, Deguchi K, Tian F, Shang J, Matsuura T and Abe K: Expression of Keap1-Nrf2 system and antioxidative proteins in mouse brain after transient middle cerebral artery occlusion. Brain Res 1370: 246-253, 2011.

48. Manickam DS, Brynskikh AM, Kopanic JL, Sorgen PL, Klyachko NL, Batrakova EV, Bronich TK and Kabanov AV: Well-defined cross-linked antioxidant nanozymes for treatment of ischemic brain injury. J Control Release 162: 636-645, 2012 . 
49. Weismann D, Hartvigsen K, Lauer N, Bennett KL, Scholl HP, Charbel Issa P, Cano M, Brandstatter H, Tsimikas S, Skerka C, et al: Complement factor $\mathrm{H}$ binds malondialdehyde epitopes and protects from oxidative stress. Nature 478: 76-81, 2011.

50. Martinic-Popovic I, Lovrencic-Huzjan A and Demarin V: Assessment of subtle cognitive impairment in stroke-free patients with carotid disease. Acta Clin Croat 48: 231-240, 2009.

51. Lee K, Kim EH, Song D, Kim YD, Nam HS, Lee HS and Heo JH: Lenticulostriate artery involvement is predictive of poor outcomes in superficial middle cerebral artery territory infarction. Yonsei Med J 58: 123-130, 2017.

52. Cahill SP, Yu RQ, Green D, Todorova EV and Snyder JS: Early survival and delayed death of developmentally-born dentate gyrus neurons. Hippocampus 27: 1155-1167, 2017.

53. Wegener S, Weber R, Ramos-Cabrer P, Uhlenkueken U, Sprenger C, Wiedermann D, Villringer A and Hoehn M: Temporal profile of T2-weighted MRI distinguishes between pannecrosis and selective neuronal death after transient focal cerebral ischemia in the rat. J Cereb Blood Flow Metab 26: 38-47, 2006.

54. Echeverry R, Wu J, Haile WB, Guzman J and Yepes M: Tissue-type plasminogen activator is a neuroprotectant in the mouse hippocampus. J Clin Invest 120: 2194-2205, 2010.

55. Miyawaki T, Ofengeim D, Noh KM, Latuszek-Barrantes A, Hemmings BA, Follenzi A and Zukin RS: The endogenous inhibitor of Akt, CTMP, is critical to ischemia-induced neuronal death. Nat Neurosci 12: 618-626, 2009.

56. Liu Y, Wang H, Liu N, Du J, Lan X, Qi X, Zhuang C, Sun T, Li Y and $\mathrm{Yu}$ J: Oxymatrine protects neonatal rat against hypoxic-ischemic brain damage via PI3K/Akt/GSK3 $\beta$ pathway. Life Sci: May 16, 2019 https://doi.org/10.1016/j.lfs.2019.04.070.

57. Cheng SM, Ho TJ, Yang AL, Chen IJ, Kao CL, Wu FN, Lin JA, Kuo $\mathrm{CH}$, Ou HC, Huang CY and Lee SD: Exercise training enhances cardiac IGFI-R/PI3K/Akt and Bcl-2 family associated pro-survival pathways in streptozotocin-induced diabetic rats. Int J Cardiol 167: 478-485, 2013.

58. Kim GD, Oh J, Park HJ, Bae K and Lee SK: Magnolol inhibits angiogenesis by regulating ROS-mediated apoptosis and the $\mathrm{PI} 3 \mathrm{~K} / \mathrm{AKT} / \mathrm{mTOR}$ signaling pathway in $\mathrm{mES} / \mathrm{EB}$-derived endothelial-like cells. Int J Oncol 43: 600-610, 2013.
59. Qi S, Xin Y, Guo Y, Diao Y, Kou X, Luo L and Yin Z: Ampelopsin reduces endotoxic inflammation via repressing ROS-mediated activation of $\mathrm{PI} 3 \mathrm{~K} / \mathrm{Akt} / \mathrm{NF}-\kappa \mathrm{B}$ signaling pathways. Int Immunopharmacol 12: 278-287, 2012.

60. Wang J, Li G, Wang Z, Zhang X, Yao L, Wang F, Liu S, Yin J, Ling EA, Wang L and Hao A: High glucose-induced expression of inflammatory cytokines and reactive oxygen species in cultured astrocytes. Neuroscience 202: 58-68, 2012.

61. Yang Y, Gozen O, Watkins A, Lorenzini I, Lepore A, Gao Y, Vidensky S, Brennan J, Poulsen D, Won Park J, et al: Presynaptic regulation of astroglial excitatory neurotransmitter transporter GLT1. Neuron 61: 880-894, 2009.

62. Mdzinarishvili A, Sumbria R, Lang D and Klein J: Ginkgo extract EGb761 confers neuroprotection by reduction of glutamate release in ischemic brain. J Pharm Pharm Sci 15: 94-102, 2012.

63. Ghosh M, Yang Y, Rothstein JD and Robinson MB: Nuclear factor- $\kappa \mathrm{B}$ contributes to neuron-dependent induction of glutamate transporter-1 expression in astrocytes. J Neurosci 31: 9159-9169, 2011.

64. Mitra R, Dube SK and Jain V: Isolated diastolic hypotension: A unique complication of intra-arterial nimodipine infusion!! J Clin Anesth 59: 1, 2020.

65. Rhee KJ, Lee CG, Kim SW, Gim DH, Kim HC and Jung BD: Extract of Ginkgo biloba ameliorates Streptozotocin-induced type 1 diabetes mellitus and High-fat diet-induced type 2 diabetes mellitus in mice. Int J Med Sci 12: 987-994, 2015.

66. Aziz TA, Hussain SA, Mahwi TO, Ahmed ZA, Rahman HS and Rasedee A: The efficacy and safety of Ginkgo biloba extract as an adjuvant in type 2 diabetes mellitus patients ineffectively managed with metformin: A double-blind, randomized, placebo-controlled trial. Drug Des Devel Ther 12: 735-742, 2018.

This work is licensed under a Creative Commons Attribution-NonCommercial-NoDerivatives 4.0 International (CC BY-NC-ND 4.0) License. 